\title{
The Importance of Precise Imaging in Villonodular Synovitis of the Hip: A Radiology Case Report
}

\author{
Andreea Belecciu, Ileana Muntean and Gellert Attila Gyurka \\ Department of Radiology, Faculty of Medicine, University of Transilvania, Brasov 500019, Romania
}

\begin{abstract}
Villonodular synovitis (VS) is a rare condition classified as a benign proliferative disorder of the synovium. An increasing number of cases describe nonspecific symptoms and diagnosis remains a challenge in corectly assessing the disease. We report the case of a 17-year-old patient that presents with persistent and worsening joint pain of the left hip, unresponsive to medication. A radiography, CT-scan (8-slice, 1G) and MRI (Philips 1 Tesla) are performed for imaging purposes. MRI findings include an intra-articular mass seen as a low to medium signal intensity heterogenous image. MRI combined with CT remains the most accurate imaging solution to diagnose such disease and particular attention is needed to correctly assess VS.
\end{abstract}

Key words: Villonodular synovitis, hip, radiology, intra-articular mass.

\section{Introduction}

Villonodular Synovitis (VS) is a rare condition in adolescents, pain of the hip in this population is extremely hard to diagnose and assess. VS is a rare benign proliferative disorder of the synovium [1], patologic aspects present as large joint effusions and erosions [2]. It has a predilection for large joints, comonly affecting the knee and hip. Diagnosis is a challenge as patients present with various symptoms, nonspecific to the disease [3-6].

We present the radiologic case of a 17-year-old woman with worsening joint pain of the left hip unresponsive to medication.

\section{Case Report}

A 17-year-old female patient presents to her primary care provider with persistent and worsening joint pain of the left hip and general myalgia, accompanied by muscle stiffness with no additional medical history. The pain does not respond to over-the-counter or anti-inflammatory analgesics, she is then sent to an orthopedic specialist. Because no concluding diagnosis is made after physical examination and analgesic

Corresponding author: Gellert Attila Gyurka, research field: radiology. treatment, she is sent to the emergency service at Spitalul Clinic Judetean de Urgenta Brasov, Romania for imaging investigations.

Patient is admitted to Spitalul Clinic Judetean de Urgenta Brasov, Romania for imaging purposes: a radiography, CT-scan (8-slice, 1G) and MRI (Philips 1 Tesla) are performed. No abnormal findings were conclusive on radiography of the hip. On CT, we can only observe rare round osteochondral lesions of $6 \mathrm{~mm}$ diameter disseminated at the femoral head and left iliac bone. The CT-scan permits exclusion of diagnosis of synovial chondromatosis as no intra-articular calcifications of the coxofemoral joint were found.

On MRI, an intra-articular mass with polycyclic outlines is observed (Fig. 1). The mass diameter average maximum is $50 \mathrm{~mm}$, seen as a low to medium signal intensity heterogeneous image in T2, T2 FS (fat saturated), STIR (short tau inversion recovery imaging), $\mathrm{T} 1$ sequences, in the posteroinferior quadrant of the left coxofemoral joint, next to the left femoral neck. Additionally, an intra-articular fluid collection on the left coxofemoral joint (Fig. 2A), with a maximum diameter of $31 \mathrm{~mm}$ and an adductor magnus and obturator externus edematous infiltration (Fig. 2B) is detected. Also, an inflammation of the left obturator 


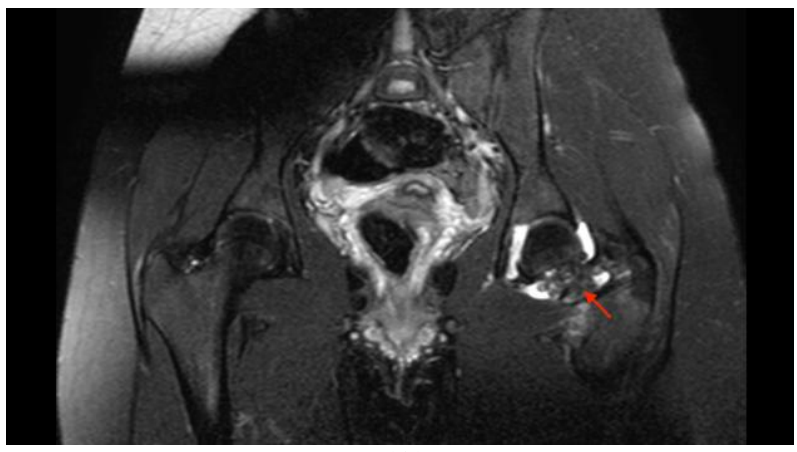

(A)

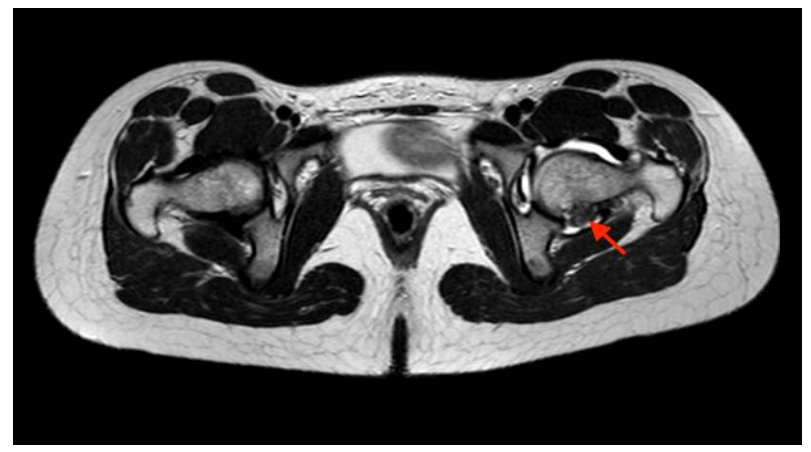

(B)

Fig. 1 (A) Coronal MRI demonstrating the characteristic low intensity intra-articular mass of villonodular synovitis on left hip (arrowhead); (B) Axial MRI demonstrating the characteristic low intensity intra-articular mass of villonodular synovitis on left hip (arrowhead).

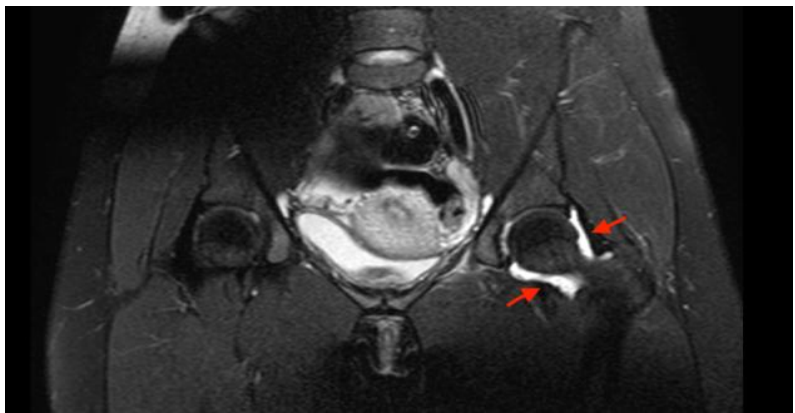

(A)

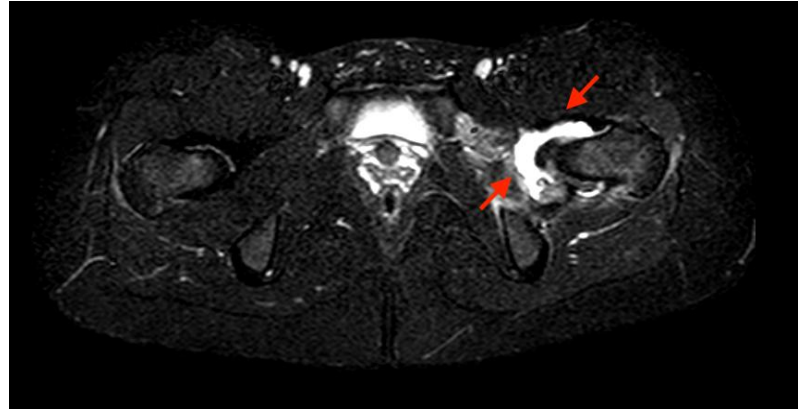

(B)

Fig. 2 (A) Coronal MRI. Intra-articular fluid collection on left coxofermoral joint (arrows); (B) Axial saturated MRI. Intra-articular fluid collection on left coxofermoral joint (arrows).

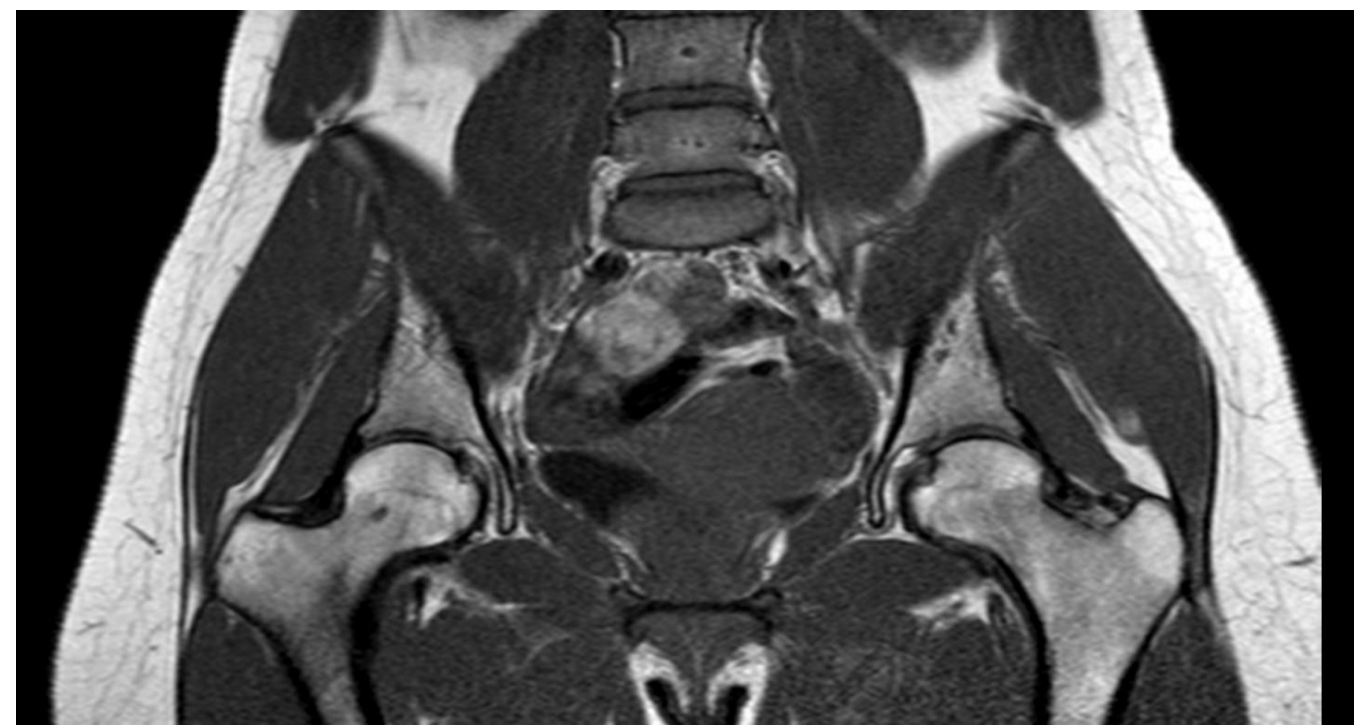

Fig. 3 Coronal MRI shows no abnormal findings.

bursa of $10 \mathrm{~mm}$ diameter is noted.

The diagnosis is confirmed by biopsy of the intra-articular piece and the patient is treated accordingly with surgery. Another MRI is performed eight months later and shows no abnormal findings and positive outcome (Fig. 3).

\section{Discussion}

The main purpose of this case is to explain the importance of precise imaging in handling patients 
with nonspecific pain at the hip joint. Diagnosis of VS remains a challenge in the medical world [4]. Patients present with various symptoms that are nonspecific to the disease [2], the disease onset is insidious and diagnostic imaging has to be reviewed accurately to find the subtle signs of VS. CT and MRI images must be obtained and scrutinized to identify VS correctly. Imaging specificity and accuracy play a great role in identifying the key elements conducting to the diagnosis.

\section{References}

[1] Lang, N., and Yuan, H-S. 2015. "Computed Tomography and Magnetic Resonance Manifestations of Spinal Pigmented Villonodular Synovitis." J. Comput. Assist. Tomogr. 39 (4): 601-6.
[2] Levy, D. M., Haughom, B. D., Nho, S. J., and Gitelis, S. 2016. "Pigmented Villonodular Synovitis of the Hip: A Systematic Review." Am. J. Orthop. 45 (1): 23-8.

[3] McCormick, F., Alpaugh, K., Haughom, B., and Nho, S. 2015. "Arthroscopic T-Capsulotomy for Excision of Pigmented Villonodular Synovitis in the Hip." Orthopedics 38 (4): 237-9.

[4] Myers, B. W., and Masi, A. T. 1980. "Pigmented Villonodular Synovitis and Tenosynovitis: A Clinical and Epidemiologic Study of 166 Cases and Literature Review." Medicine (Baltimore). 59 (3): 223-38.

[5] True, V. L., Monsell, F. P., Smith, T. A., and Steward, C. G. 2015. "A Severe Systemic Presentation of Pigmented Villonodular Synovitis in a Child with Underlying Chediak-Higashi Syndrome." Journal of Pediatric Orthopaedics B 24: 526-9.

[6] Zara, C., and Botez, P. 2009. "Osteonecrosis of the Femoral Head-Differential Diagnosis." Jurnalul de Chirurgie, Iasi 5 (4): 344-8. (in Romanian) 